\title{
Computational identification and experimental validation of microRNAs binding to the Alzheimer-related gene ADAM10
}

Regina Augustin ${ }^{1}$, Kristina Endres², Sven Reinhardt ${ }^{2}$ Peer-Hendrik Kuhn ${ }^{3}$, Stefan F Lichtenthaler ${ }^{3}$, Jens Hansen ${ }^{1}$, Wolfgang Wurst ${ }^{1,3,4^{*}}$ and Dietrich Trümbach ${ }^{1,4^{*}}$

\begin{abstract}
Background: MicroRNAs (miRNAs) are post-transcriptional regulators involved in numerous biological processes including the pathogenesis of Alzheimer's disease (AD). A key gene of AD, ADAM10, controls the proteolytic processing of APP and the formation of the amyloid plaques and is known to be regulated by miRNA in hepatic cancer cell lines. To predict miRNAs regulating ADAM10 expression concerning AD, we developed a computational approach.

Methods: MiRNA binding sites in the human ADAM10 3' untranslated region were predicted using the RNA22, RNAhybrid and miRanda programs and ranked by specific selection criteria with respect to AD such as differential regulation in AD patients and tissue-specific expression. Furthermore, target genes of miR-103, miR-107 and miR-1306 were derived from six publicly available miRNA target site prediction databases. Only target genes predicted in at least four out of six databases in the case of miR-103 and miR-107 were compared to genes listed in the AlzGene database including genes possibly involved in AD. In addition, the target genes were used for Gene Ontology analysis and literature mining. Finally, we used a luciferase assay to verify the potential effect of these three miRNAs on ADAM10 3'UTR in SH-SY5Y cells.

Results: Eleven miRNAs were selected, which have evolutionary conserved binding sites. Three of them (miR-103, miR-107, miR-1306) were further analysed as they are linked to $A D$ and most strictly conserved between different species. Predicted target genes of miR-103 ( $p$-value $=0.0065)$ and miR-107 ( $p$-value $=0.0009)$ showed significant overlap with the AlzGene database except for miR-1306. Interactions between miR-103 and miR-107 to genes were revealed playing a role in processes leading to AD. ADAM10 expression in the reporter assay was reduced by miR-1306 (28\%), miR-103 (45\%) and miR-107 (52\%).

Conclusions: Our approach shows the requirement of incorporating specific, disease-associated selection criteria into the prediction process to reduce the amount of false positive predictions. In summary, our method identified three miRNAs strongly suggested to be involved in AD, which possibly regulate ADAM10 expression and hence offer possibilities for the development of therapeutic treatments of AD.
\end{abstract}

\footnotetext{
*Correspondence: wurst@helmholtz-muenchen.de; dietrich.truembach@ helmholtz-muenchen.de

${ }^{1}$ Helmholtz Centre Munich, German Research Centre for Environmental Health $(\mathrm{GmbH})$ and Technical University Munich, Institute of Developmental Genetics, Ingolstädter Landstraße. 1, 85764 Munich-Neuherberg, Germany ${ }^{3}$ DZNE-German Center for Neurodegenerative Diseases, Schillerstrasse 44, 80336 Munich, Germany

Full list of author information is available at the end of the article
} 


\section{Background}

MicroRNAs (miRNAs) are on average 22 nucleotides long and play a pivotal role in gene regulation. These small RNAs regulate the gene expression posttranscriptionally by suppression of mRNA translation, stimulation of mRNA deadenylation and degradation or induction of target mRNA cleavage, but have also the potential to activate translation [1,2]. Over half of the mammalian protein coding-genes are regulated by miRNAs and most human mRNAs have binding sites for miRNAs [3]. The interaction of miRNA and target mRNA requires base pairing between the seed sequence (positions 2-8) of the miRNA at the $5^{\prime}$ end and a sequence most frequently found in the $3^{\prime}$ untranslated region (UTR) of the target mRNA [4]. MiRNAs are involved in neuronal functions like neurite outgrowth and brain development. They were recently described to play a role in human neurodegenerative diseases. Changes in miRNA expression profiles or miRNA target sequences could contribute to the development of Parkinson's disease and Alzheimer's disease (AD) $[5,6]$.

Characteristics of $\mathrm{AD}$ are insoluble plaques of amyloid $\beta(A \beta)$ peptides emerging from the cleavage of the amyloid beta precursor protein $(A P P)$ and neuro-fibrillary tangles in the brains of $\mathrm{AD}$ patients $[7,8]$. The alphasecretase "a disintegrin and metalloproteinase 10" (ADAM10) [9-11] generates soluble secreted amyloid precursor protein-alpha (sAPP $\alpha$ ) and avoids formation of plaques, because it cleaves $A P P$ inside the $A \beta$ sequence [12].

Numerous available computational methods predict a large number of genes targeted by miRNAs regulating gene expression, but only few have been validated experimentally. Many computational predictions are false positives and therefore have to be filtered out [13]. The requirement of target-site conservation in different species including far related species would be a potential way to reduce the false positive rate [14].

In this study we established an approach to identify miRNAs regulating ADAM10 expression which therefore might influence the progression of AD. The three programs RNA22, RNAhybrid and miRanda predicted potential miRNA binding sites to ADAM10. We sought to identify the most interesting miRNAs possibly binding to $A D A M 10$ with additional selection criteria in particular whether they play a role in AD. Additionally, the most interesting miRNAs were experimentally verified by a luciferase assay. Our results show that $m i R-103$, $m i R-107$ and $m i R-1306$ influence the expression of $A D A M 10$ at least in the reporter assay system. These miRNAs could play a role in $\mathrm{AD}$ and therefore are interesting candidates to be further analysed concerning their biological function and relation to AD.

\section{Methods}

\section{miRNA target site prediction databases}

MiRNA binding sites to target genes were downloaded from seven different databases: miRBase, 5-Nov-2007, http://www.mirbase.org/ [15]; microRNA, September 2008 Release, http://www.microrna.org/microrna/home. do [16]; PicTar via UCSC Table Browser, assembly = May 2004 $(\mathrm{NCBI} 35 / \mathrm{hg} 17)$, group = Regulation, track = PicTar miRNA, http://genome.ucsc.edu/ [17]; PITA, version 6 (31-Aug-2008), http://genie.weizmann.ac.il/pubs/ mir07/index.html [18]; RNA22, March 2007, http:// cbcsrv.watson.ibm.com/rna22.html [19]; TarBase, June 2008, http://diana.cslab.ece.ntua.gr/tarbase/ [20]; TargetScan, Release 5, http://www.targetscan.org/ [3]. We established a workflow considering all miRNA target site predictions downloaded.

\section{miRNA target prediction}

We used three prediction programs RNA22, RNAhybrid, miRanda and predicted all binding sites of the miRNA sequences to the 3'UTR sequence of human ADAM10.

RNA22 is a pattern-based method for the identification of miRNA-target sites. The method has high sensitivity, is resilient to noise, can be applied to the analysis of any genome without requiring genome-specific retraining and does not rely upon cross-species conservation. Focusing on novel features of miRNA-mRNA interaction RNA22 first finds putative miRNA binding sites in the sequence of interest then identifies the targeting miRNA and hence allows to identify sites targeted by yet-undiscovered miRNAs. An implementation of RNA22 (19-May-2008) is available online at http:// cbcsrv.watson.ibm.com/rna22.html [19,21].

The second program RNAhybrid is an extension of the classical RNA secondary structure prediction algorithm from Zuker and Stiegler [22]. It finds the energetically most favorable hybridization sites of a small RNA in a large RNA incorporating 'seed-match speed-up', which first searches for seed matches in the candidate targets and only upon finding such matches the complete hybridization around the seed-match is calculated. The user can define the position and length of the seed region with the option to allow for G:U wobble base pairs in the seed pairing. Intramolecular base pairings and branching structures are forbidden and statistical significance of predicted targets is assessed with an extreme value statistics of length normalized minimum free energies, a Poisson approximation of multiple binding sites, and the calculation of effective numbers of orthologous targets in comparative studies of multiple organisms. RNAhybrid, Version 2.1, is available online at http://bibiserv.techfak.uni-bielefeld.de/rnahybrid/ [23,24]. 
The miRanda algorithm is similar to the SmithWaterman algorithm, but scores based on the complementarity of nucleotides $(A=U$ or $\mathrm{G} \equiv \mathrm{C})$ and one $\mathrm{G}: \mathrm{U}$ wobble pair is allowed in the seed region but has to be compensated by matches in the $3^{\prime}$ end of miRNA. In order to estimate the thermodynamic properties of a predicted pairing between miRNA and 3 UTR sequence, the algorithm uses folding routines from the Vienna 1.3 RNA secondary structure programming library (RNAlib) [25]. A conservation filter is used and optionally some rudimentary statistics about each target site can be generated. MiRanda, September 2008 Release, is available online at http://www.microrna.org/microrna/home.do $[21,26]$.

The parameter setting for RNA22 is: maximum number of "UN-paired" bases within the extent of the seed = 0 , extent of seed in nucleotides $=6$, minimum number of paired-up bases that you want to see in any reported heteroduplex $=14$, maximum value for the folding energy in any reported heteroduplex $=-25 \mathrm{kcal} / \mathrm{mol}$. The parameter setting for RNAhybrid is: "-s 3utr_human" (“-s" tells RNAhybrid to quickly estimate statistical parameters from "minimal duplex energies" under the assumption that the target sequences are human $3^{\prime} U T R$ sequences). The parameter setting for miRanda is the default parameter setting: gap open penalty $=-8$, gap extend $=-2$, score threshold $=50$, energy threshold $=$ $-20 \mathrm{kcal} / \mathrm{mol}$, scaling parameter $=4$.

We retrieved the $3^{\prime} \mathrm{UTR}$ sequence of $A D A M 10$ (human ADAM10 3'UTR based on transcript NM_001110 (chr15:58888510-58889745)) from NCBI http://www. ncbi.nlm.nih.gov/. We downloaded 703 mature miRNA sequences for Homo sapiens from miRBase, version 13.0 http://www.mirbase.org/ [15].

\section{Extraction of best miRNA predictions}

The extraction of miRNAs was applied according to the following selection criteria. We checked for each miRNA how many programs predicted the miRNA to bind to human ADAM10 $3^{\prime} \mathrm{UTR}$. The regulation of miRNAs in $\mathrm{AD}$ was verified by the publication of Cogswell et al. [27], which provides a list of miRNAs expressed in the tissues hippocampus, cerebellum and medial frontal gyrus. Another possibility to check the expression of miRNAs in the brain is the Mouse Genome Informatics (MGI) database (Mouse Genome Database, The Jackson Laboratory, Bar Harbor, Maine; http://www.informatics. jax.org/) [28]. Literature search by PubMed was done as an additional approval, to search for already described target genes of the miRNAs, especially for target genes involved in AD. Mouse ADAM10 3'UTR based on transcript NM_007399 (chr9:70625902-70628036) from NCBI http://www.ncbi.nlm.nih.gov/ was used for binding site search of mouse miRNAs from miRBase, version
13.0 http://www.mirbase.org/ [15]. The parameter setting for RNA22 and miRanda is the same as for human miRNA binding site prediction at the human ADAM10 3'UTR. The parameter setting for RNAhybrid is "-d $1.9,0.28$ " (1.9 is the location parameter and 0.28 the shape parameter of the assumed extreme value distribution). Additionally, we searched by TargetScan database http://www.targetscan.org/ [3] and microRNA database http://www.microrna.org/microrna/home.do [16] for miRNAs binding to human ADAM10 3'UTR and compared the TargetScan and microRNA predictions to our list of miRNAs for equal miRNAs. We identified the number of binding sites of a miRNA in the human ADAM10 3'UTR predicted by each program. ADAM10 $3^{\prime}$ UTR sequences from ten different species were analysed for conserved regions. The following sequences where taken: human ADAM10 3'UTR from transcript NM_001110 (chr15:58888510-58889745), mouse ADAM10 3'UTR from transcript NM_007399 (chr9:70625902-70628036), horse ADAM10 3'UTR from transcript XM_001498169.1 (chr1:132875124-132876868), dog ADAM10 3'UTR from transcript XM_858910 (chr30:26596273-26598436), chimp ADAM10 3'UTR from transcript XM_001172393.1 (chr15:55942343-55944774), chicken ADAM10 3'UTR from transcript ENSGALT00000034458 (chr10:79497687951846), rhesus monkey ADAM10 3'UTR from transcript XM_001096908 (chr7:36929437-36932008), zebra fish $A D A M 10$ 3'UTR from transcript NM_001159314 (chr7:31745579-31747655), opossum ADAM10 3'UTR from transcript ENSMODT00000011088 (chr1:162230000162230183), zebra finch $A D A M 103^{\prime} \mathrm{UTR}$ from transcript XR_054746 (chr10:6638729-6639273). For multiple sequence alignment of the ten ADAM10 3'UTR sequences we applied ClustalW Version 2.1 from the European Bioinformatics Institute (EBI) http://www.ebi. ac.uk/ $[29,30]$. We used default parameters except: DNA Weight Matrix = 'ClustalW', Clustering = 'UPGMA'. After extraction of the conserved regions between at least seven species we looked for miRNA binding sites localized in these conserved regions. Additionally, we determined the conservation (given in percentage) of the miRNA binding site sequence from human to each species.

\section{Statistical analysis}

Statistical analysis was performed with $\mathrm{R}$ statistical software ( $\mathrm{R}$ 2.8.0, http://www.r-project.org/). The $p$-value was computed by the $\mathrm{R}$ function fisher.test with default settings. The Fisher's exact test is used to examine the significance of the association (contingency) between the two kinds of classification. Significantly regulated genes were considered, if the $p$-value is equal or below 0.05 . We generated Venn diagrams to see the overlap between target genes of miR-103 and miR-107 common in 4 out 
of 6 databases as well as genes in the AlzGene database (http://www.alzgene.org/; Version: 20.06.2011) [31]. Each set of target genes of $m i R-103$ and $m i R-107$ common in 4 out of 6 databases as well as the set of target genes of miR-1306 in the database PITA was explored for enrichment in Gene Ontology [32] by the software Pathway Studio 8.0 (Ariadne Genomics) based on database ResNet 8.0.

\section{Literature mining and pathway analysis}

Literature search by PubMed was done to extract information about the target genes of the miRNAs resulting from Pathway Studio analysis and their relation to AD. To verify the miRNAs searches were performed for miRNA interactions in all PubMed abstracts with the help of the text mining program Pathway Studio 8.0 (Ariadne Genomics) based on the Natural Language Processing (NLP) Technology. Pathway analysis was done with the software Ingenuity Systems IPA 9.0 (http://www.ingenuity.com/) especially with the Path Designer.

\section{Material}

Mature miRNAs and the inactive negative control were from Invitrogen (No. PM11012, PM13206, PM10632, PM10056). All RNA species were dissolved to $5 \mathrm{pmol} / \mu \mathrm{l}$ in nuclease-free water upon arrival, aliquoted and stored at $-20^{\circ} \mathrm{C}$.

\section{Cloning of the ADAM10 3'UTR luciferase reporter construct}

The 3'UTR of human ADAM10 was amplified from THP-1 chromosomal DNA using the FailSafe PCR kit (Epicentre) and the following primers:

\section{AD10_3UTR_for 5'GCGGCCGCGCCCATTCAGCA ACCCCAG $3^{\prime}$ \\ AD10_3UTR_rev 5'GCGGCCGCCACTTGTGCCCG TAGCAGCC 3 .}

The obtained DNA fragment was verified by restriction digestion and sequencing. The $3^{\prime} \mathrm{UTR}$ was subsequently cloned into the NotI site of the pCMV-GLuc vector (NEB), which allows to monitor regulated Gaussia luciferase expression in the cell supernatant.

\section{Cell culture}

SH-SY5Y cells were cultivated in phenol red-free DMEM/F12, supplemented with 10\% FCS and 1\% glutamine at $37^{\circ} \mathrm{C}, 95 \%$ air moisture, $5 \% \mathrm{CO}_{2}$ and passaged twice a week with a splitting rate of $1 / 2$ to $1 / 4$.

\section{3'UTR luciferase reporter assay}

Retro-transfection was performed using $0.005 \mu$ l Lipofectamine 2000 (Invitrogen) per $\mu$ l OptiMEM-medium and $0.1 \mathrm{pmol} / \mu \mathrm{l}$ miRNA (Invitrogen) or negative control. For combination of miRNA 1306 together with miRNA 103 or 107 a concentration of $0.05 \mathrm{pmol} / \mu \mathrm{l}$ each was used. $2 \mathrm{ng} / \mu \mathrm{l}$ endotoxin-free plasmid DNA of the 3'UTR-reporter vector were added to 45.000 cells per well in 96 well format. Control cells were mock-treated with nuclease-free water instead of RNA molecules.

$5 \mathrm{hrs}$ after transfection, the cell supernatant was exchanged to $200 \mu \mathrm{l}$ culture medium per well. In a preliminary experiment, $10 \mu \mathrm{l}$ cell supernatant were collected at various time points over a 72 hour period; 48 hours were determined to be the optimal incubation time (data not shown). Therefore, $10 \mu$ l cell supernatant were aspirated 48 hours after transfection and stored at $-20^{\circ} \mathrm{C}$ until samples were measured. Secreted Gaussia luciferase was quantitatively analyzed (Renilla-Luciferase assay, Promega) using the FluostarOptima luminometer (BMG). Cell densities were checked by quantitation of protein content in the cell lysate by NanoQuant assay (Roth).

\section{Results and discussion \\ MiRNA prediction}

We established a workflow (Figure 1) to obtain miRNAs possibly binding to the human $A D A M 103^{\prime} \mathrm{UTR}$. Instead of looking up miRNA target sites of $A D A M 10$ in the miRNA target site prediction databases, we predicted the binding sites of human miRNAs to human ADAM10 3'UTR by the three programs, RNA22, RNAhybrid and miRanda, with the aim to yield a more accurate miRNA target site prediction. Due to the fact that the three prediction programs focus on different aspects for miRNA target site prediction (pattern-based search, seed matching, conservation, energy or structure) various properties of the target sequence are covered (see Methods: "miRNA target prediction").

122 miRNAs are predicted by at least two programs to bind to human $A D A M 10$ 3'UTR sequence and 52 of them are significant according to expression and selection criteria described in the following. To consider different aspects of the distinct prediction algorithms at least two programs should predict a miRNA binding site. Important is also the expression of the miRNA in brain provided by the MGI database or the regulation of the miRNA in AD as described by Cogswell and colleagues (2008) in the tissues hippocampus, cerebellum and medial frontal gyrus [27]. An additional confirmation of miRNA being involved in $\mathrm{AD}$ is a binding site to a target gene, which is involved in $\mathrm{AD}$, described in the literature and thus the miRNA might regulate also other $A D$ key genes such as ADAM10. Furthermore, the miRNA 


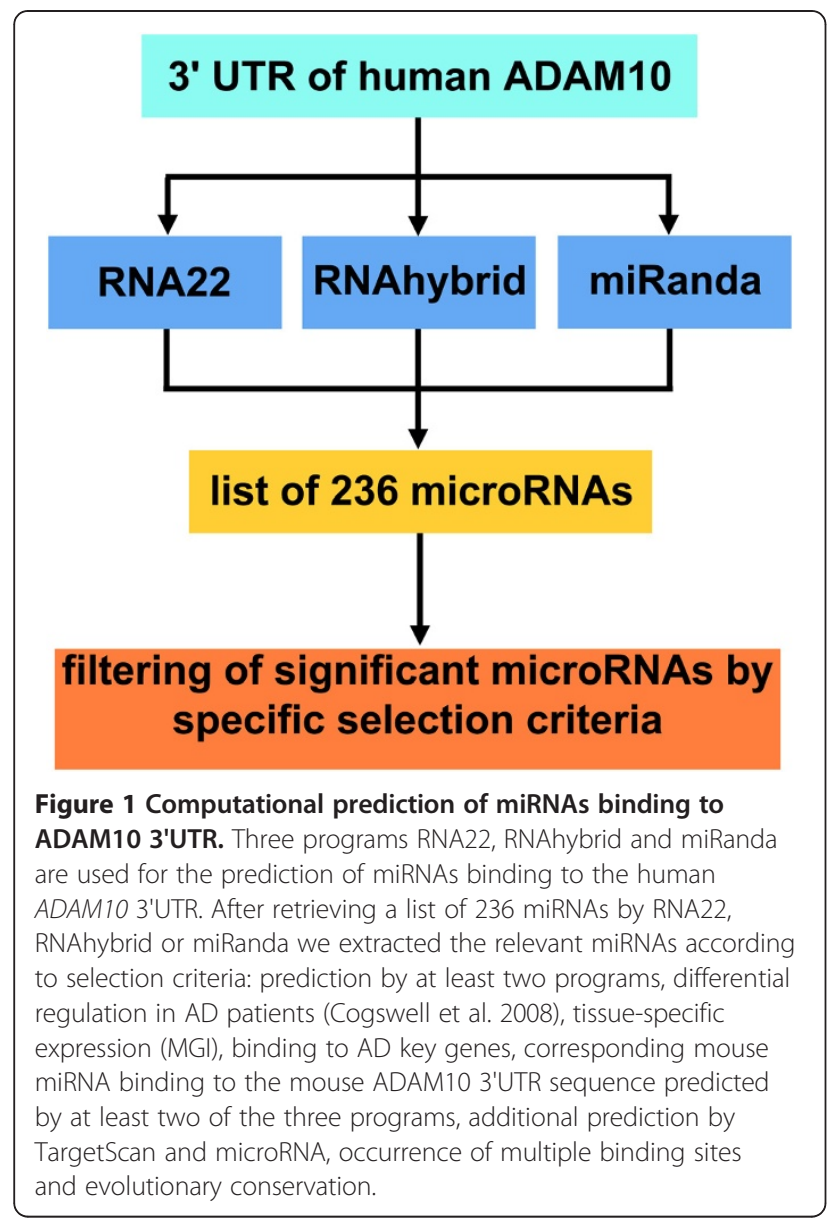

prediction is strengthened by the corresponding mouse miRNA binding to the mouse ADAM10 3'UTR sequence predicted by at least two of the three miRNA prediction programs RNA22, RNAhybrid and miRanda. Prediction of the miRNA by other webtools such as TargetScan and microRNA is also a confirmation of the miRNA. Multiple binding sites of a single miRNA in the 3'UTR verify the prediction [33].

The list of 52 miRNAs incorporates not conserved as well as conserved miRNAs or rather miRNA binding sites. Filtering according to conservation helps to reduce the number of miRNAs and improves the selection of candidates for further experimental validations. Consideration of conservation across species, including those not developing $\mathrm{AD}$, was chosen as a filter criterion because a correlation between miRNA conservation and disease susceptibility has in general been suggested by $\mathrm{Lu}$ et al. [34]. Our selection procedure therefore excludes non-conserved miRNA binding sites, which also might be relevant for development of the disease but are not lost by being included in the list of $52 \mathrm{miR}$ NAs. In regard to $\mathrm{AD}$ being a human disease, future analysis of non-conserved miRNAs might also represent a valuable approach which we did not follow in the context of this manuscript. In our analysis eleven miRNA binding sites are conserved across at least seven species, at which four of these miRNAs are also conserved in the far related species zebra fish. The only miRNA binding site miR-1306 in the human ADAM10 $3^{\prime}$ UTR predicted by all three programs is also conserved in the far related zebra fish as well as in mouse, horse, dog, chimp, chicken, rhesus monkey and zebra finch (Figure 2A). This binding site is located on chromosome 15 positions 58889309-58889324 and the programs RNA22, RNAhybrid and miRanda predicted the binding energy -32.29 , -25.7 and $-22.55 \mathrm{kcal} / \mathrm{mol}$, respectively. The conservation of the miRNA binding site sequence between human and the species mouse, horse, dog, chimp as well as rhesus monkey is $100 \%$. The conservation of chicken, zebra finch and zebra fish to human in this binding region is $94 \%, 88 \%$ and $75 \%$, respectively. The second most interesting miRNAs possibly binding to human ADAM10 3'UTR are miR-103 as well as miR107 both having the same binding site located on chromosome 15 positions 58889443-58889468. This site is predicted by the two programs RNAhybrid and miRanda with binding energy -27.9 and $-23.66 \mathrm{kcal} / \mathrm{mol}$ for $m i R-103$, respectively, as well as -26.2 and $-22.28 \mathrm{kcal} / \mathrm{mol}$ for $m i R-107$, respectively. The conservation of the miRNA binding site sequence between human and the species mouse, horse, dog and chimp is $96 \%$, while the conservation of chicken, rhesus monkey and finch to human is $65 \%, 100 \%$ and $73 \%$, respectively (Figure 2B-C). Additionally, miR-202, miR-423-5p, miR503, $m i R-184$ and $m i R-922$ bind also to the conserved binding region chromosome 15 positions 588894435889473 and $m i R-330-5 p$ (chr15:58889149-58889178), miR-671-5p (chr15:58889720-58889745) and miR-432 (chr15:58889688-58889718) bind to a region with good conservation also to the far related species zebra fish, but these eight miRNAs have no indication to be involved in AD (Table 1). Table 1 shows a ranked list of the best miRNA binding site predictions according to the specific selection criteria. We chose the three most interesting miRNAs 1306, 107 and 103 and performed analyses with the AlzGene database, further miRNA target site prediction databases, Gene Ontology, literature mining and validation experiments to identify the involvement in AD. MiR-107 and miR-103 are downregulated with age [35] as well as in AD gray matter [36] and repress the translation of cofilin. In brains of a transgenic mouse model of AD the level of $m i R-103$ and $m i R-107$ is decreased while the cofilin protein level is increased which results in the formation of rod-like structures [37]. Furthermore, $m i R-107$ expression is decreased even in the earliest stages of AD. As $m i R-107$ regulates beta-site $\mathrm{APP}$-cleaving enzyme 1 (BACE1) it might be involved in accelerated disease progression 


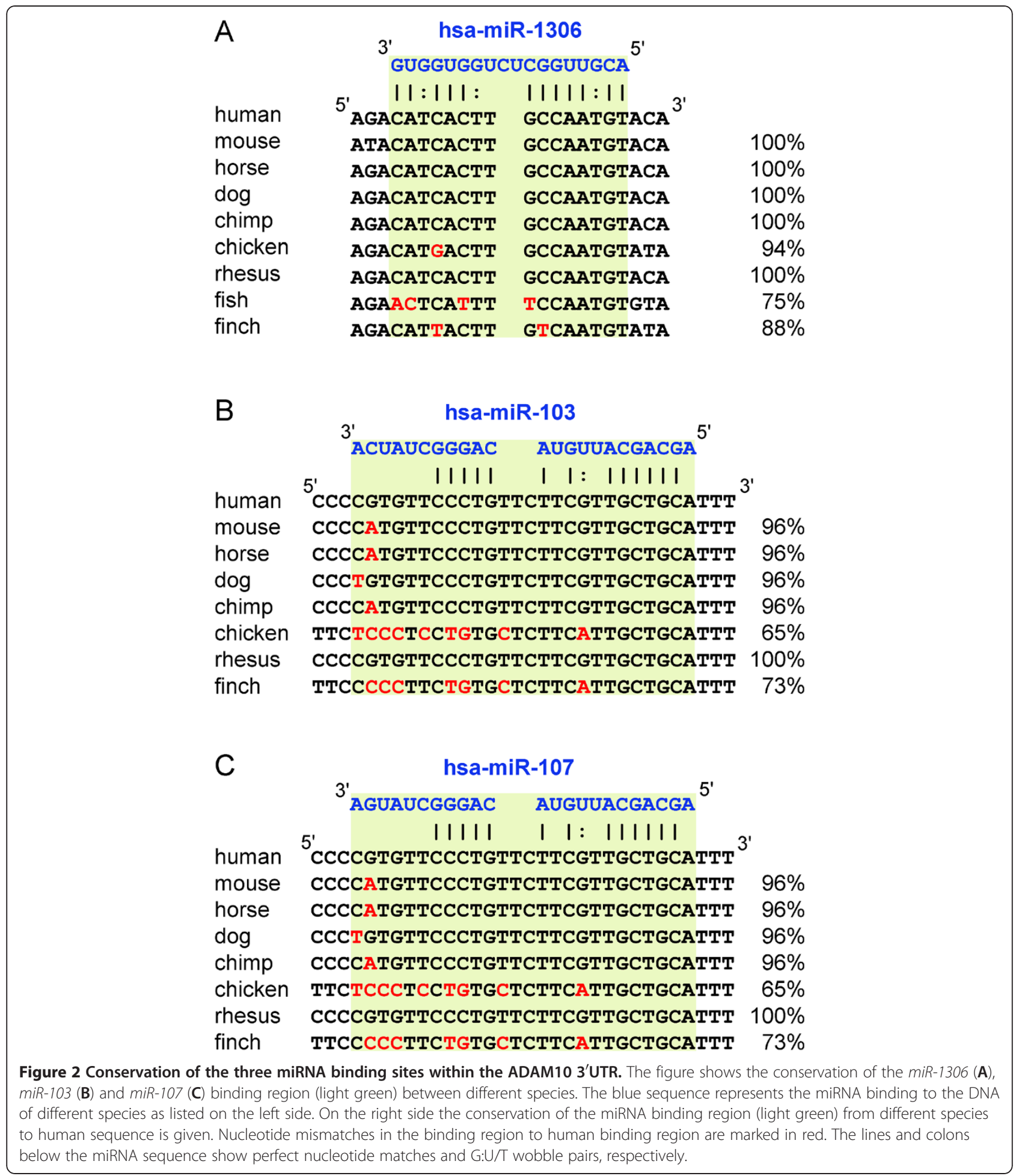

[38]. The downregulation of miR-103 and miR-107 with age could concern a protective effect against plaque formation because reduced levels of these miRNAs would lead to an increased level of the predicted target ADAM10 and its neuroprotective product sAPP $\alpha$ in brains of $\mathrm{AD}$ patients. Our observations of strong inhibition (> 40\%) of ADAM10 expression in the reporter assay upon application of miR-103 and miR-107 would coincide with such a possible protective influence on amyloid pathology (see "Experimental validation of bioinformatically predicted miRNAs"). According to the publication from Cogswell et al. [27] $m i R-103$ is 
Table 1 List of predicted miRNAs binding to a conserved region of human ADAM10 3'UTR

\begin{tabular}{|c|c|c|c|c|}
\hline miRNA & $\emptyset \mathrm{kcal} / \mathrm{mol}$ & Confirmations & Differential regulated in $A D$ & $\begin{array}{l}\text { Conservation } \\
\text { zebra fish }\end{array}$ \\
\hline 1306 & -26.85 & predicted by 3 programs, mouse ADAM 10 & & + \\
\hline 107 & -24.24 & $\begin{array}{l}\text { targets BACE1, predicted by TargetScan, literature for AD, } \\
\text { mouse ADAM10 }\end{array}$ & & \\
\hline 103 & -25.78 & predicted by TargetScan, literature for AD, mouse ADAM10 & hippocampus, cerebellum & \\
\hline $330-5 p$ & -27.20 & predicted by microRNA, mouse ADAM10 & hippocampus & + \\
\hline 432 & -22.81 & predicted by microRNA & cerebellum & + \\
\hline $423-5 p$ & -22.1 & mouse ADAM10 & hippocampus, medial frontal gyrus & \\
\hline $671-5 p$ & -27.61 & mouse ADAM10 & & + \\
\hline 922 & -27.99 & predicted by microRNA & & \\
\hline 503 & -25.41 & predicted by microRNA, mouse ADAM10 & & \\
\hline 202 & -25.33 & predicted by microRNA, mouse ADAM10 & & \\
\hline 184 & -23.33 & mouse ADAM10 & & \\
\hline
\end{tabular}

The best eleven predicted miRNAs, which bind to a conserved region of human ADAM10 3'UTR, are shown with additional information like the average predicted binding energy in $\mathrm{kcal} / \mathrm{mol}$ (column 2), verifications of the miRNAs from literature, by additional mouse ADAM10 prediction or other prediction programs (column 3), the regulation of miRNAs in AD according to Cogswell et al. (column 4) and the conservation of the binding region in zebra fish marked with + in the last column.

differentially expressed in hippocampus and cerebellum in AD. In addition, the program TargetScan verifies the same binding site of miR-103 and miR-107 to human ADAM10. MiR-1306 is further analysed due to its good conservation to the far related species zebra fish with only one mismatch in the seed region. It is the only miRNA whose binding site to human ADAM10 is predicted by all three programs RNA22, RNAhybrid and miRanda, which strengthens the assumption that this binding site is functionally active. Additionally as shown in Figure 3 the hypothesis is verified that miR-1306 is associated to AD: Twelve predicted target genes of miR-1306 are involved in processes and functions playing a role in $\mathrm{AD}$, the nervous system and other neurodegenerative diseases. The predictions rely on TargetScan while the dedicated functions are Ingenuity Expert Findings or from Gene Ontology. MiR-1306 possibly regulates genes like the cholinergic receptor, nicotinic, alpha 4 (CHRNA4), tumor necrosis factor receptor superfamily member $1 \mathrm{~B}$ (TNFRSF1B) and mitogenactivated protein kinase kinase $4(M A P 2 K 4)$, which are associated to $\mathrm{AD}$ by the functions frontotemporal dementia, demyelination of neurons and Huntington's disease, respectively. MAP $2 K 4$ has been found to be involved in $\mathrm{AD}$ and is putatively regulated by modules of transcription factor binding sites [39]. Furthermore, miR-1306 is located on chromosome 22 within the second exon of DiGeorge syndrome critical region gene

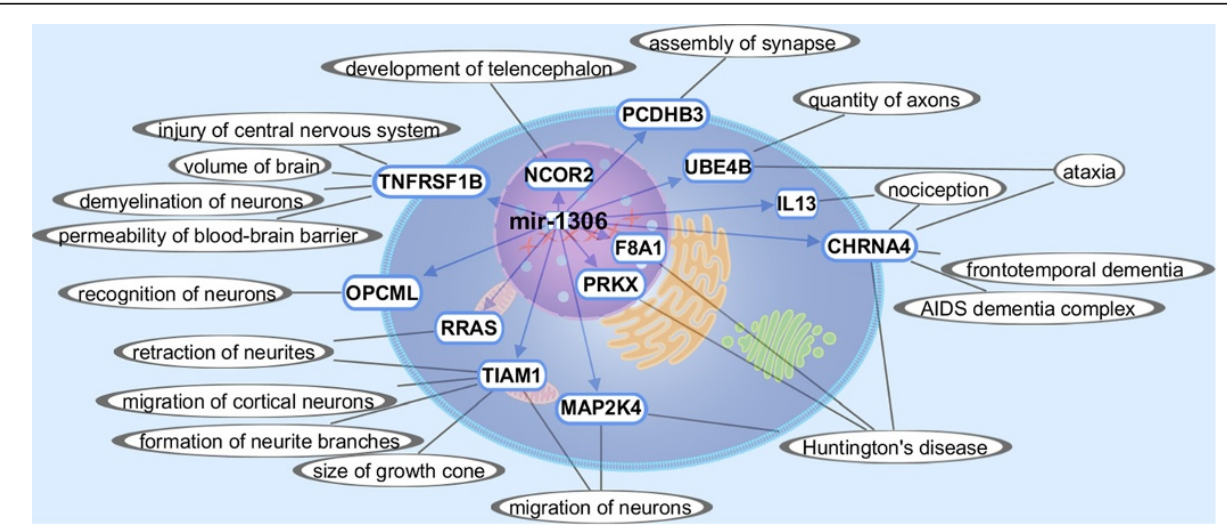

Figure 3 Target gene predictions for miR-1306. Twelve target genes of miR-1306 were predicted by TargetScan concerning their function in $A D$, brain, nervous system or other neurodegenerative diseases and graphically interrelated by Path Designer (Ingenuity). The miRNA is located in the nucleus and the predicted target genes indicated by blue arrows and blue framed ellipses are located according to their Gene Ontology either in the nucleus, cytoplasm or membrane. The functions of the target genes are denoted by grey lines and grey framed ellipses and derived from Gene Ontology or Ingenuity Expert Findings, which are substantiated by literature. 
8 (DGCR8), which is essential for miRNA biogenesis by being a subunit of the microprocessor complex [40]. Evers et al. presents a case of a DiGeorge syndrome patient with the typical deletion in chromosome band 22q11.2, which contains DGCR8, suffering from dementia [41].

Prediction of miRNA target genes and their relation to AD To confirm the relationship of the three miRNAs to AD we searched for target gene predictions of these miRNAs in the six databases miRBase, microRNA, PicTar, PITA, RNA22, as well as TargetScan (Figure 4). The combination of different miRNA target site prediction databases and the restriction of the output to four out of six databases in the case of $m i R-103$ and $m i R-107$ are important to reduce the amount of false positive miRNA target sites in the end. In the case of $m i R-1306$ there is no restriction of the output, because only the database PITA incorporates predictions for this miRNA. Additionally, Tarbase can be included in the analysis. The database Tarbase has a special position, because in contrast to the other six databases Tarbase contains experimentally supported miRNA targets and not in-silico predicted miRNA binding sites. All miRNA target sites from Tarbase are automatically included in the output of the analysis. In contrast to the other six databases Tarbase doesn't contain false positive miRNA target sites. In our case Tarbase doesn't contain target genes for the miRNAs: $m i R-103, m i R-107$ and $m i R-1306$. The six miRNA target site prediction databases miRBase, microRNA, PicTar, PITA, RNA22, and TargetScan contain altogether 18915 different (according to EntrezGene) human genes at which PITA alone contains 16819 genes. After the analysis we got 156 and 157 target genes for miR-103 and $m i R-107$, respectively, common in four out of six databases, and 890 target genes for $m i R-1306$ of database

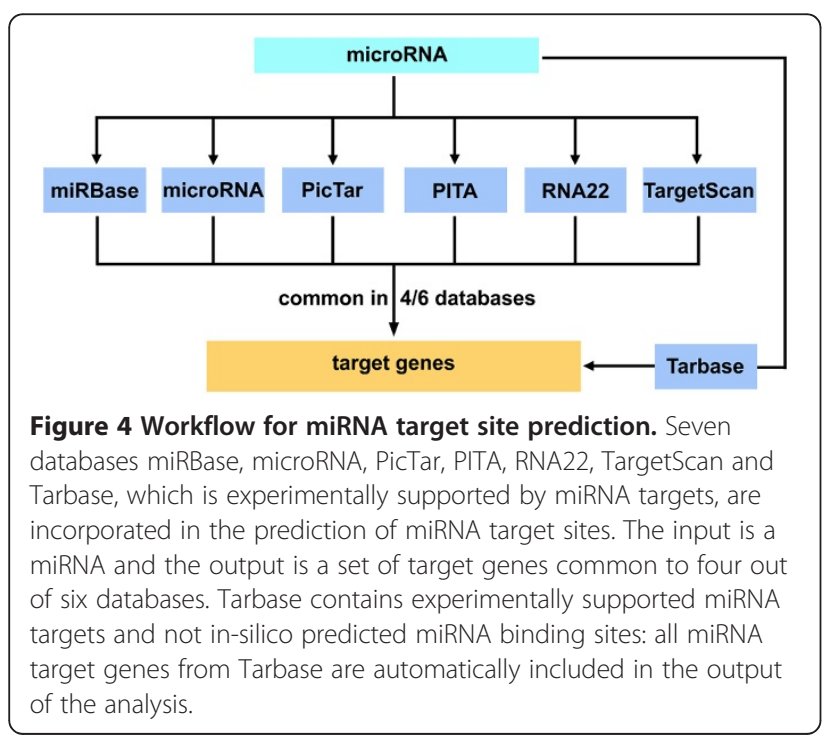

PITA. As we focus on miRNAs playing a role in $\mathrm{AD}$, we used the AlzGene database to see which target genes of the miRNAs have a genetic association with AD. AlzGene database is a regularly updated aggregation of all published genetic association studies including GWAS (genome-wide association studies) performed on AD phenotypes. 636 and 591 genes of AlzGene database overlap with 18915 genes of the six miRNA target site prediction databases and 16819 genes of database PITA, respectively. The overlap between AlzGene database genes and the target genes of $m i R-103, m i R-107$ as well as $m i R-1306$ are 12, 14 (Figure 5) and 24 genes, respectively (Additional file 1 ). MiR-103 and $m i R-107$ have 130 target genes in common (Figure 5, Additional file 1). Applying a Fisher's exact test we got a $p$-value of 0.0065 , 0.0009 and 0.1904 for the overlap of $m i R-103, m i R-107$ and $m i R-1306$, respectively, with the AlzGene database, which shows that 12 and 14 are significant high numbers of overlapping genes between the target genes and the AlzGene database. This result suggests that miR-103 and miR-107 might play a role in AD. It is not remarkable that the $p$-value for the overlap of $m i R-1306$ with the AlzGene database is not significant as the restriction to four out of six databases was not possible, which consequentially leads to the inclusion of a lot of false positive target genes in the 890 target genes of $m i R-1306$.

\section{Gene ontology}

Additionally, we did a Gene Ontology analysis with the predicted target genes of the three miRNAs to validate the functionality of the miRNAs and their involvement

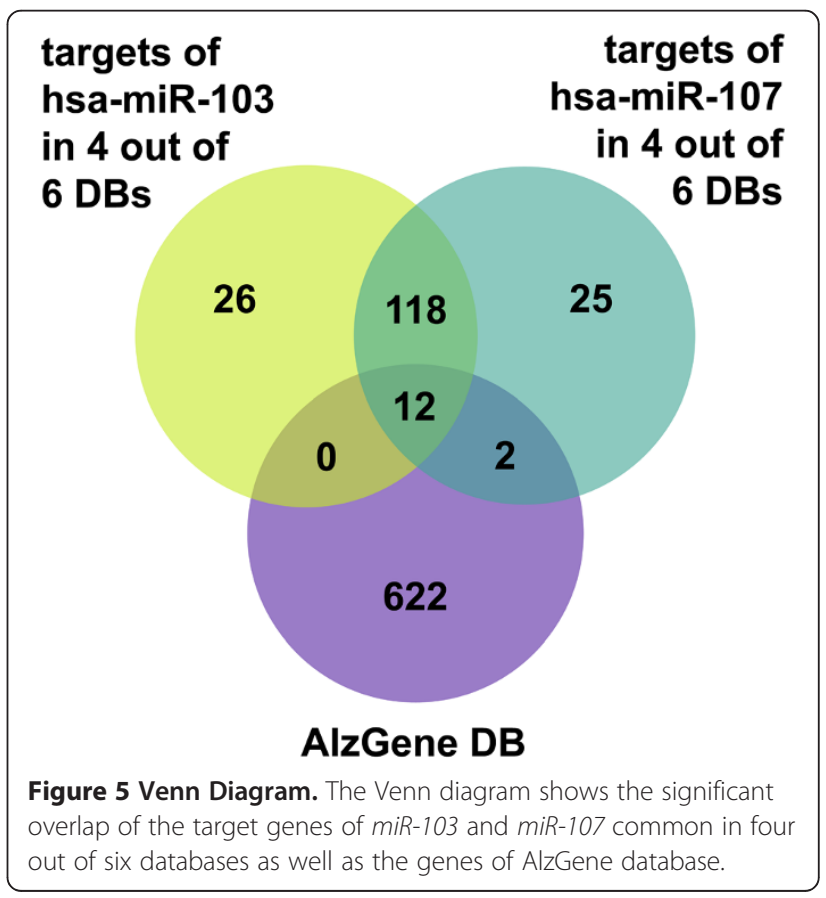


to AD. With the help of the literature mining tool Pathway Studio we searched for molecular functions and biological processes common to the target genes of the three miRNAs. The molecular function calcium ion binding is significant in all three miRNA analyses (miR103: $p$-value $=0.0011 ;$ miR-107: $p$-value $=0.0025 ;$ miR1306: $p$-value $=2.1 \times 10^{-5}$ ) and is considered to be involved in $\mathrm{AD}$. Calcium ions are found in an elevated level in tangle-bearing neurons of AD patients compared to healthier neurons [42]. Further, an abnormal increase of intracellular Calcium ion levels in neurites associated with $A \beta$ deposits was demonstrated in a mouse model of $\mathrm{AD}$ [43]. An additional evidence is given by the significant biological processes learning $(m i R-103: p$-value $=$ $0.0008 ; \quad m i R-107: \quad p$-value $=3.3 \times 10^{-5} ; \quad m i R-1306: p$ value $=0.0003)$, brain development $(m i R-103: p$-value $=$ $0.0023 ; m i R-107: p$-value $=0.0004 ; m i R-1306: p$-value $=$ $\left.7.1 \times 10^{-6}\right)$ and nervous system development (miR-103: $p$-value $=0.0004 ; \quad m i R$-107: $p$-value $=0.0004 ; \quad m i R-1306$ : $p$-value $=6.9 \times 10^{-8}$ ) that the three miRNAs are involved in AD. Mouse models with an overexpression of $A D A M 10$ showed a positive effect of the $\alpha$-secretase on learning and memory and mice with a dominant negative mutant form of $A D A M 10$ had learning deficiencies [12]. Environmental influences occurring during brain development predefine the expression and regulation of APP. As a consequence levels of APP and A $\beta$ are increased causing $A D$ later in life [44]. The $A \beta$ fragments forming plaques are of varying length depending on the site of cleavage. The $A \beta_{42}$ fragment is a ligand for the cellular prion protein, which is important for nervous system development [45]. (See Additional files 2, 3 and 4: List of enriched target genes of miR-103/107/ 1306 in Gene Ontology).

\section{Literature mining}

Furthermore, a network (Figure 6) containing already published interactions of $m i R-103$ and $m i R-107$ with genes involved in AD or included in the AlzGene database was established using the literature mining tool Pathway Studio. This network allowed confirming the relation of the two miRNAs to $\mathrm{AD}$ by their respective target genes. The genes dicer 1 , ribonuclease type III (Dicer) and TAR (HIV-1) RNA binding protein 2 (TARBP2) targeted by miRNA-103 and miRNA-107 are components of the miRNA-processing complex [46]. Besides those two genes, a link between the two miRNAs is provided by linoleic acid [47], which probably affects AD by increasing the expression of Presenilin1 (PSEN1) and $\mathrm{A} \beta$ [48]. Another target gene in the network, Granulin $(G R N)$, is regulated by miR-107 [49] which regulates $B A C E 1$ as well [38]. Therefore both genes might be involved in neurodegenerative diseases especially AD. The tumor suppressors TP53 as well as TP73 appear to regulate the processing of $m i R-107$ [46]. MiR-103 increases the expression level of fatty acid binding protein 4 (FABP4) while its expression is reduced by tumor necrosis factor (TNF) [50]. All six target genes of the miRNAs 103 and 107 GRN, BACE1, TP53, TP73, FABP4 and $T N F$ are included in the AlzGene database, hence putatively playing a role in $\mathrm{AD}$. The four remaining target genes cyclin-dependent kinase 2 (CDK2), cAMP responsive element binding protein 1 (CREB1), nuclear factor I/A (NFIA) and vascular endothelial growth factor A (VEGFA) of the network are mentioned in literature to be involved directly or in processes developing AD. miR-103 directly binds and represses CDK2 and CREB1 through $3^{\prime} U T R$ binding [51]. CDK2 is a key regulator in neuronal differentiation with the downregulation of $C D K 2$ as crucial event [52] and neuronal differentiation is regulated by PSEN1, a major key gene of $\mathrm{AD}$ [53]. The transcription factor $C R E B 1$ is involved in several types of learning and memory. A direct involvement in $\mathrm{AD}$ is seen in some mouse models, where its activity is impaired [54]. NFIA is negatively regulated by $m i R-107$ [55] and plays an important role in the formation of the corpus callosum in the developing brain. The disruption of NFIA results in agenesis of the corpus callosum [56], whereas the size of the corpus callosum is significantly reduced in AD patients [57]. The expression of the hypoxia-regulated gene VEGFA is decreased by $m i R-107$ [58]. Additionally, it is known, that polymorphisms within the VEGFA promoter region are associated with increased risk for $\mathrm{AD}$, by reducing the neuroprotective effect of VEGFA [59]. These findings of the literature and AlzGene database confirm the biological role of the target genes in neurodegenerative processes and hence the involvement of $m i R-103$ and $m i R-107$ in AD.

\section{Experimental validation of bioinformatically predicted miRNAs}

To demonstrate that the selected miRNAs 1306, 103 and 107 directly regulate $A D A M 10$ expression by interaction with the $3^{\prime} U T R$ of the human gene, we performed cotransfection experiments with a Gaussia reporter vector harbouring the $3^{\prime} \mathrm{UTR}$ of $A D A M 10$ downstream of the luciferase coding sequence together with the respective miRNAs. As a positive control we used $m i R$ 122 which has been identified and validated as an important regulator of $A D A M 10$ in hepatocellular carcinoma by an experimental approach [60]. The programs RNA22, RNAhybrid and miRanda predicted a miR-122 binding site to human ADAM10 3'UTR with binding energy $-31.2,-25.6$ and $-21.84 \mathrm{kcal} / \mathrm{mol}$ (on average: $-26.21 \mathrm{kcal} / \mathrm{mol}$ ), respectively, comparable to the miR-1306 binding site prediction (see Results and Discussion: "miRNA prediction"). Time resolved measurement revealed that $48 \mathrm{hrs}$ incubation period resulted 


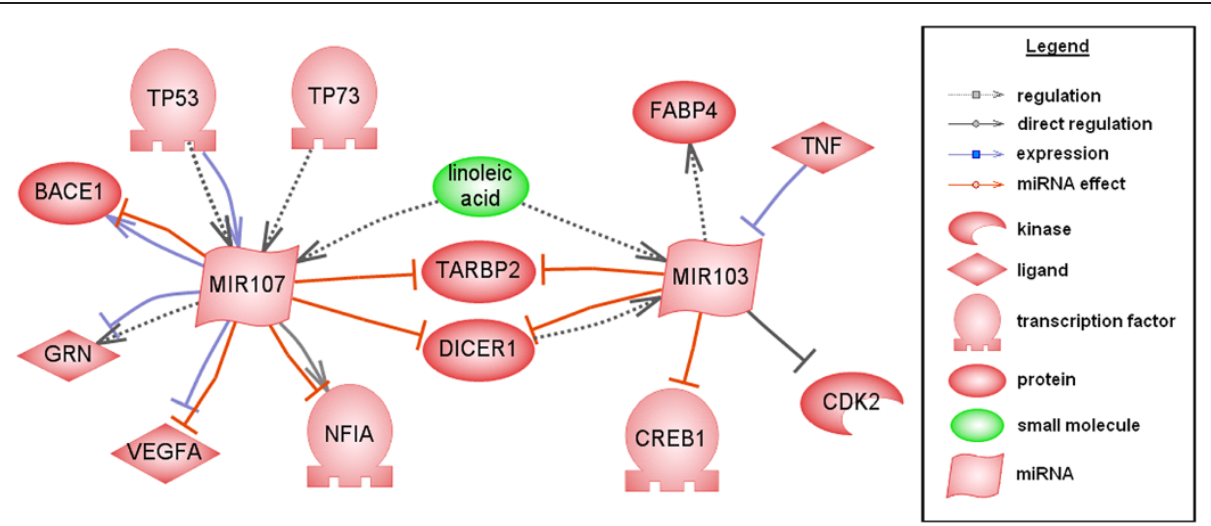

Figure 6 Interaction network of miR-103 and miR-107. The network (established by Pathway Studio) shows already published interactions between miR-103 as well as miR-107 and genes known to be involved in AD or neurodegenerative processes. The different types of proteins or small molecules are indicated by different symbols and the various interactions like regulation, expression and miRNA effect are also displayed by various arrows.

in maximal effects of the miRNAs in SH-SY5Y cells (data not shown): while the negative control miRNA had no impact on luciferase activity measured in the cell supernatant, miR-122 reduced the luminescent signal to $57 \%$ as compared to water treated control cells (Figure 7). This effect is even higher than the one observed in the initial publication from Bai et al. (2009)

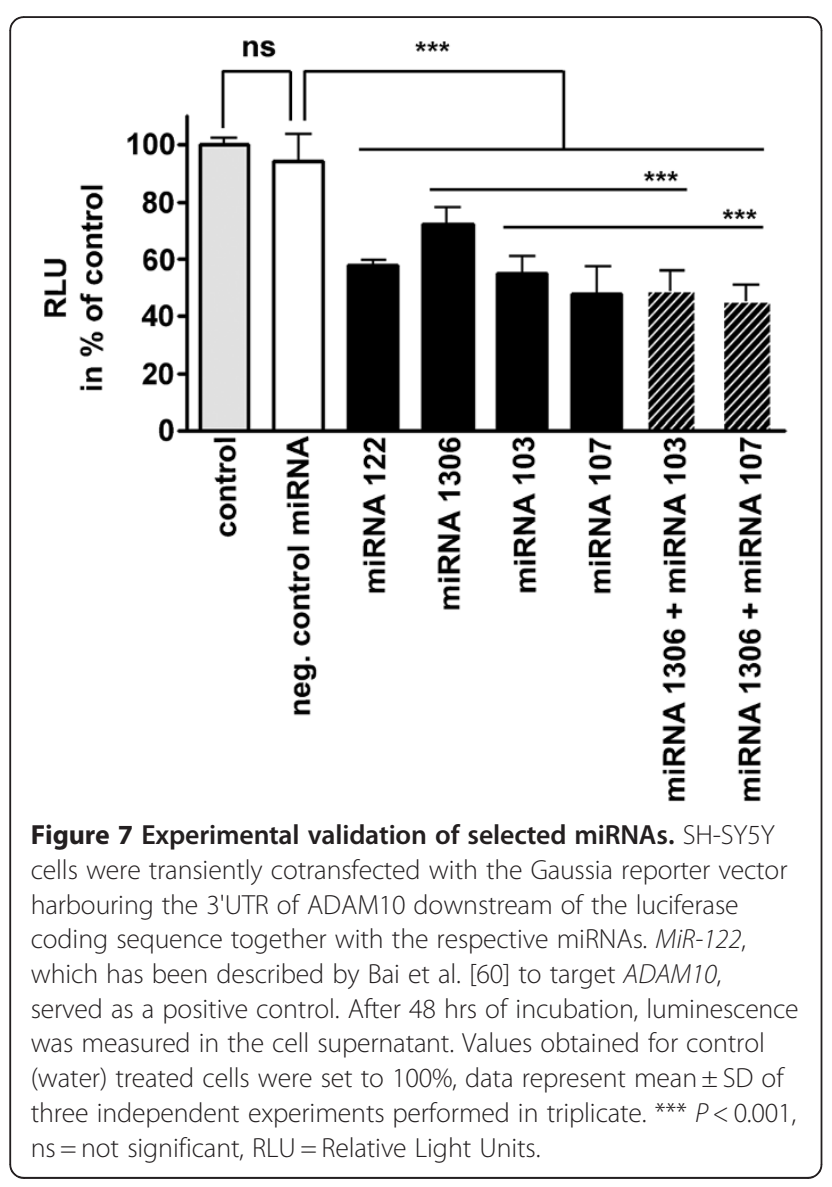

[60] but might be due to the different reporter enzymes or cell lines used. The three miRNAs identified by bioinformatical approaches and integration of literature mining all showed a significant decreasing effect on the ADAM10 3'UTR-reporter construct: miR-1306 lowered the luminescent signal to $72 \%, m i R-103$ to $55 \%$ and $m i R$ 107 to $48 \%$ of control. While miR-103 and miR-107 target the same DNA sequence within the ADAM10 3'UTR (see Figure 2), miR-1306 has a binding site in closer proximity to the Stop codon. We therefore combined miR-1306 and miR-103 or miR-107, respectively, but observed no distinct significant synergistic effect (miR1306 vs. $m i R-1306 / 103 p<0.001$; miR-1306 vs. miR$1306 / 107 p<0.001)$. This might be due to the applied concentration which was reduced to $50 \%$ in the combinations (see methods " 3 'UTR luciferase reporter assay"). These experimental results suggest an influence of $m i R$ 103, miR-107 and miR-1306 on ADAM10 expression. Nevertheless, the biological impact of either miRNA has to be elucidated further, e.g. by mRNA and protein measurements. Assessing the effect of the selected miRNAs on pathological features in $\mathrm{AD}$ mouse models would also help to understand their distinct role in pathogenesis. However, this study shows that the computational workflow consisting of prediction programs and specific selection criteria is a suitable tool for the identification of miRNAs influencing key genes of diseases such as Alzheimer's disease.

\section{Conclusions}

We established a computational approach for the identification of miRNAs putatively influencing the expression of $A D A M 10$. A potential functionality of selected miRNAs 103, 107 and 1306 was confirmed by 3'UTR luciferase reporter assay. These results show that the evolutionary conservation of the target gene binding site 
facilitates miRNA candidate selection independently from the disease for further experimental validation. Moreover, these experiments underline the reliability of our computational approach, which is a combination of characteristics of the prediction software and specific selection criteria for filtering out false positive predictions: disease relevance, specificity of expression, evolutionary conservation of binding sites and occurrence of multiple binding sites. This workflow can also be applied to key genes of other diseases with adjustment of the selection criteria according to the scientific research interest. Our approach provides a new selection tool for identification and ranking of $\mathrm{AD}$-related miRNAs, but to elucidate a profound pathological role of selected candidates, further experiments have to be done.

\section{Additional files}

Additional file 1: List of predicted target genes common in 4 out of 6 DBs. The table shows a list of predicted target genes of miR-103, miR-107, miR-1306. These target genes are either listed in AlzGene DB and target gene of at least one miRNA or target gene of at least two miRNAs (column 3). Beside the gene symbol the Entrez GenelD is given.

Additional file 2: List of enriched target genes of miR-103 in Gene Ontology. The table shows the Gene Ontology entities with enrichment of predicted target genes of miR-103. Additionally a $p$-value and FDR value is given.

Additional file 3: List of enriched target genes of miR-107 in Gene Ontology. The table shows the Gene Ontology entities with enrichment of predicted target genes of miR-107. Additionally a $p$-value and FDR value is given.

Additional file 4: List of enriched target genes of miR-1306 in Gene Ontology. The table shows the Gene Ontology entities with enrichment of predicted target genes of miR-1306. Additionally a $p$-value and FDR value is given.

\section{Abbreviations \\ Chr: Chromosome; Human: Homo sapiens; Mouse: Mus musculus; Horse: Equus caballus; Dog: Canis lupus familiaris; Chimp: Pan troglodytes; Chicken: Gallus gallus; Rhesus monkey: Macaca mulatta; Zebra fish: Danio rerio; Opossum: Monodelphis domestica; Zebra finch: Taeniopygia guttata.}

\section{Competing interests}

The authors declare that they have no competing interests.

\section{Authors' contributions}

RA performed the bioinformatic analyses and wrote the manuscript. RA and DT designed the computational workflow. KE designed the experimental system. SR performed transfection and luciferase reporter assay experiments. SFL and PHK contributed to the planning of the whole project. JH participated in the conception of this work. WW directed the work. DT planed and coordinated the entire project. All authors read and approved the final manuscript.

\section{Acknowledgements}

We thank Bernd Lentes for technical assistance. This work was supported by the Federal Ministry of Education and Research (BMBF) in the framework of the National Genome Research Network (NGFN), Förderkennzeichen FKZ01GS08130, by the BMBF Project "Kompetenznetzwerk Demenzen Neurodegeneration" (KNDD, FKZ 01 G1 704) to SFL and WW, by the BMBF Project "Helmholtz Alliance for Mental Health in an Ageing Society" (HelMA, HA215), by the Deutsche Forschungsgemeinschaft (DFG) Project SFB 596 TP A 12 and by the NGFN Project (FKZ 01GS08133).

\section{Author details}

'Helmholtz Centre Munich, German Research Centre for Environmental Health $(\mathrm{GmbH})$ and Technical University Munich, Institute of Developmental Genetics, Ingolstädter Landstraße. 1, 85764 Munich-Neuherberg, Germany. ${ }^{2}$ Department of Psychiatry and Psychotherapy, University Medical Centre of the Johannes Gutenberg-University Mainz, Untere Zahlbacher Str. 8, 55131 Mainz, Germany. ${ }^{3}$ DZNE-German Center for Neurodegenerative Diseases, Schillerstrasse 44, 80336 Munich, Germany. ${ }^{4}$ Max Planck Institute of Psychiatry, Kraepelinstr. 2-10, 80804 Munich, Germany.

Received: 22 December 2011 Accepted: 19 April 2012

Published: 17 May 2012

\section{References}

1. Chekulaeva M, Filipowicz W: Mechanisms of miRNA-mediated posttranscriptional regulation in animal cells. Curr Opin Cell Biol 2009, 21:452-460

2. Vasudevan S, Steitz JA: AU-rich-element-mediated upregulation of translation by FXR1 and Argonaute 2. Cell 2007, 128:1105-1118.

3. Friedman RC, Farh KK-H, Burge CB, Bartel DP: Most mammalian mRNAs are conserved targets of microRNAs. Genome Res 2009, 19:92-105.

4. Fabian MR, Sonenberg N, Filipowicz W: Regulation of mRNA translation and stability by microRNAs. Annu Rev Biochem 2010, 79:351-379.

5. Hébert SS, De Strooper B: Alterations of the microRNA network cause neurodegenerative disease. Trends Neurosci 2009, 32:199-206.

6. Satoh J: MicroRNAs and their therapeutic potential for human diseases: aberrant microRNA expression in Alzheimer's disease brains. J Pharmacol Sci 2010, 114:269-275.

7. Crews L, Rockenstein E, Masliah E: APP transgenic modeling of Alzheimer's disease: mechanisms of neurodegeneration and aberrant neurogenesis. Brain Struct Funct 2010, 214:111-126.

8. Cole SL, Vassar R: The role of amyloid precursor protein processing by BACE1, the beta-secretase, in Alzheimer disease pathophysiology. J Biol Chem 2008, 283:29621-29625.

9. Lammich S, Kojro E, Postina R, Gilbert S, Pfeiffer R, Jasionowski M, Haass C, Fahrenholz F: Constitutive and regulated alpha-secretase cleavage of Alzheimer's amyloid precursor protein by a disintegrin metalloprotease. Proc Natl Acad Sci 1999, 96:3922-3927.

10. Kuhn PH, Wang H, Dislich B, Colombo A, Zeitschel U, Ellwart JW, Kremmer E, Rossner S, Lichtenthaler SF: ADAM10 is the physiologically relevant, constitutive alpha-secretase of the amyloid precursor protein in primary neurons. EMBO J 2010, 29:3020-3032.

11. Lichtenthaler SF, Haass C, Steiner H: Regulated intramembrane proteolysis - lessons from amyloid precursor protein processing. J Neurochem 2011, 117:779-796.

12. Postina R, Schroeder A, Dewachter I, Bohl J, Schmitt U, Kojro E, Prinzen C, Endres K, Hiemke C, Blessing M, Flamez P, Dequenne A, Godaux E, van Leuven F, Fahrenholz F: A disintegrin-metalloproteinase prevents amyloid plaque formation and hippocampal defects in an Alzheimer disease mouse model. J Clin Invest 2004, 113:1456-1464.

13. Watanabe $Y$, Tomita M, Kanai A: Computational methods for microRNA target prediction. Methods Enzymol 2007, 427:65-86.

14. Lewis $B P$, Burge $C B$, Bartel DP: Conserved seed pairing, often flanked by adenosines, indicates that thousands of human genes are microRNA targets. Cell 2005, 120:15-20.

15. Griffiths-Jones S, Saini HK, van Dongen S, Enright AJ: miRBase: tools for microRNA genomics. Nucleic Acids Res 2008, 36:D154-D158.

16. Betel D, Wilson M, Gabow A, Marks DS, Sander C: The microRNA.org resource: targets and expression. Nucleic Acids Res 2008, 36:D149-D153.

17. Krek A, Grün D, Poy MN, Wolf R, Rosenberg L, Epstein EJ, MacMenamin P, da Piedade I, Gunsalus KC, Stoffel M, Rajewsky N: Combinatorial microRNA target predictions. Nat Genet 2005, 37:495-500.

18. Kertesz M, lovino N, Unnerstall U, Gaul U, Segal E: The role of site accessibility in microRNA target recognition. Nat Genet 2007, 39:1278-1284.

19. Miranda KC, Huynh T, Tay Y, Ang Y-S, Tam W-L, Thomson AM, Lim B, Rigoutsos I: A pattern-based method for the identification of MicroRNA binding sites and their corresponding heteroduplexes. Cell 2006, 126:1203-1217.

20. Papadopoulos GL, Reczko M, Simossis VA, Sethupathy P, Hatzigeorgiou AG: The database of experimentally supported targets: a functional update of TarBase. Nucleic Acids Res 2009, 37:D155-D158. 
21. Witkos TM, Koscianska E, Krzyzosiak WJ: Practical Aspects of microRNA Target Prediction. Curr Mol Med 2011, 11:93-109.

22. Zuker M, Stiegler P: Optimal computer folding of large RNA sequences using thermodynamics and auxiliary information. Nucleic Acids Res 1981, 9:133-148.

23. Rehmsmeier M, Steffen $P$, Hochsmann M, Giegerich R: Fast and effective prediction of microRNA/target duplexes. RNA 2004, 10:1507-1517.

24. Krüger J, Rehmsmeier M: RNAhybrid: microRNA target prediction easy, fast and flexible. Nucleic Acids Res 2006, 34:W451-W454.

25. Wuchty S, Fontana W, Hofacker IL, Schuster P: Complete suboptimal folding of RNA and the stability of secondary structures. Biopolymers 1999, 49:145-165.

26. Enright AJ, John B, Gaul U, Tuschl T, Sander C, Marks DS: MicroRNA targets in Drosophila. Genome Biol 2003, 5:R1

27. Cogswell JP, Ward J, Taylor IA, Waters M, Shi Y, Cannon B, Kelnar K, Kemppainen J, Brown D, Chen C, Prinjha RK, Richardson JC, Saunders AM, Roses AD, Richards CA: Identification of miRNA changes in Alzheimer's disease brain and CSF yields putative biomarkers and insights into disease pathways. J Alzheimers Dis 2008, 14:27-41

28. Bult CJ, Eppig JT, Kadin JA, Richardson JE, Blake JA, Mouse Genome Database Group: The Mouse Genome Database (MGD): mouse biology and model systems. Nucleic Acids Res 2008, 36:D724-D728.

29. Larkin MA, Blackshields G, Brown NP, Chenna R, McGettigan PA, McWilliam H, Valentin F, Wallace IM, Wilm A, Lopez R, Thompson JD, Gibson TJ, Higgins DG: Clustal W and Clustal X version 2.0. Bioinformatics 2007, 23:2947-2948

30. Goujon M, McWilliam H, Li W, Valentin F, Squizzato S, Paern J, Lopez R: A new bioinformatics analysis tools framework at EMBL-EBI. Nucleic Acids Res 2010, 38:W695-W699.

31. Bertram L, McQueen MB, Mullin K, Blacker D, Tanzi RE: Systematic metaanalyses of Alzheimer disease genetic association studies: the AlzGene database. Nat Genet 2007, 39:17-23.

32. Ashburner M, Ball CA, Blake JA, Botstein D, Butler $H$, Cherry JM, Davis AP, Dolinski K, Dwight SS, Eppig JT, Harris MA, Hill DP, Issel-Tarver L, Kasarskis A, Lewis S, Matese JC, Richardson JE, Ringwald M, Rubin GM, Sherlock G: Gene Ontology: tool for the unification of biology. Nat Genet 2000, 25:25-29.

33. Sethupathy P, Megraw M, Hatzigeorgiou AG: A guide through present computational approaches for the identification of mammalian microRNA targets. Nat Meth 2006, 3:881-886.

34. Lu M, Zhang Q, Deng M, Miao J, Guo Y, Gao W, Cui Q: An analysis of human microRNA and disease associations. PLoS One 2008, 3:e3420

35. Noren Hooten N, Abdelmohsen $K$, Gorospe M, Ejiogu N, Zonderman AB, Evans MK: microRNA expression patterns reveal differential expression of target genes with age. PLoS One 2010, 5:e10724.

36. Wang WX, Huang Q, Hu Y, Stromberg AJ, Nelson PT: Patterns of microRNA expression in normal and early Alzheimer's disease human temporal cortex: white matter versus gray matter. Acta Neuropathol 2011, 121:193-205.

37. Yao J, Hennessey T, Flynt A, Lai E, Beal MF, Lin MT: MicroRNA-related cofilin abnormality in Alzheimer's disease. PLoS One 2010, 5:e15546.

38. Wang WX, Rajeev BW, Stromberg AJ, Ren N, Tang G, Huang Q, Rigoutsos t. Nelson PT: The expression of microRNA miR-107 decreases early in Alzheimer's disease and may accelerate disease progression through regulation of beta-site amyloid precursor protein-cleaving enzyme 1 . J Neurosci 2008, 28:1213-1223.

39. Augustin R, Lichtenthaler SF, Greeff M, Hansen J, Wurst W, Trümbach D: Bioinformatics identification of modules of transcription factor binding sites in Alzheimer's disease-related genes by in silico promoter analysis and microarrays. International Journal of Alzheimer's Disease 2011, 2011:154325.

40. Wang $Y$, Medvid R, Melton C, Jaenisch R, Blelloch R: DGCR8 is essential for microRNA biogenesis and silencing of embryonic stem cell self-renewal. Nat Genet 2007, 39:380-385.

41. Evers LJ, Vermaak MP, Engelen JJ, Curfs LM: The velocardiofacial syndrome in older age: dementia and autistic features. Genet Couns 2006, 17:333-340

42. Nixon RA, Saito Kl, Grynspan F, Griffin WR, Katayama S, Honda T, Mohan PS, Shea TB, Beermann M: Calcium-activated neutral proteinase (calpain) system in aging and Alzheimer's disease. Ann N Y Acad Sci 1994, 747:77-91.
43. Mattson MP: ER calcium and Alzheimer's disease: in a state of flux. Sci Signal 2010, 3:pe10.

44. Zawia NH, Lahiri DK, Cardozo-Pelaez F: Epigenetics, oxidative stress, and Alzheimer disease. Free Radic Biol Med 2009, 46:1241-1249.

45. Kim D, Tsai LH: Bridging physiology and pathology in AD. Cell 2009, 137:997-1000

46. Boominathan $L$ : The tumor suppressors $p 53, p 63$, and $p 73$ are regulators of microRNA processing complex. PLoS One 2010, 5:e10615.

47. Parra P, Serra F, Palou A: Expression of adipose microRNAs is sensitive to dietary conjugated linoleic acid treatment in mice. PLoS One 2010, 5:e13005.

48. Liu Y, Yang L, Conde-Knape K, Beher D, Shearman MS, Shachter NS: Fatty acids increase presenilin-1 levels and gamma-secretase activity in PSwt-1 cells. J Lipid Res 2004, 45:2368-2376.

49. Wang WX, Wilfred BR, Madathil SK, Tang G, Hu Y, Dimayuga J, Stromberg AJ, Huang Q, Saatman KE, Nelson PT: miR-107 regulates granulin/progranulin with implications for traumatic brain injury and neurodegenerative disease. Am J Pathol 2010, 177:334-345.

50. Xie H, Lim B, Lodish HF: MicroRNAs induced during adipogenesis that accelerate fat cell development are downregulated in obesity. Diabetes 2009, 58:1050-1057.

51. Liao Y, Lönnerdal B: Global microRNA characterization reveals that miR103 is involved in IGF-1 stimulated mouse intestinal cell proliferation. PLoS One 2010, 5:e12976.

52. Dobashi Y, Kudoh T, Matsumine A, Toyoshima K, Akiyama T: Constitutive overexpression of CDK2 inhibits neuronal differentiation of rat pheochromocytoma PC12 cells. J Biol Chem 1995, 270:23031-23037.

53. Wines-Samuelson $M$, Handler $M$, Shen J: Role of presenilin- 1 in cortical lamination and survival of Cajal-Retzius neurons. Dev Biol 2005, 277:332-346.

54. Puzzo D, Vitolo O, Trinchese F, Jacob JP, Palmeri A, Arancio O: Amyloidbeta peptide inhibits activation of the nitric oxide/cGMP/CAMPresponsive element-binding protein pathway during hippocampal synaptic plasticity. J Neurosci 2005, 25:6887-6897.

55. Garzon R, Pichiorri F, Palumbo T, Visentini M, Ageilan R, Cimmino A, Wang H, Sun H, Volinia S, Alder H, Calin GA, Liu CG, Andreeff M, Croce CM: MicroRNA gene expression during retinoic acid-induced differentiation of human acute promyelocytic leukemia. Oncogene 2007, 26:4148-4157.

56. Das Neves L, Duchala CS, Godinho F, Haxhiu MA, Colmenares C, Macklin WB, Campbell CE, Butz KG, Gronostajski RM: Disruption of the murine nuclear factor I-A gene (Nfia) results in perinatal lethality, hydrocephalus, and agenesis of the corpus callosum. Proc Natl Acad Sci 1999, 96:11946-11951.

57. Teipel SJ, Bayer W, Alexander GE, Zebuhr Y, Teichberg D, Kulic L, Schapiro MB, Moller H-J, Rapoport SI, Hampel H: Progression of corpus callosum atrophy in Alzheimer disease. Arch Neurol 2002, 59:243-248.

58. Yamakuchi M, Lotterman CD, Bao C, Hruban RH, Karim B, Mendell JT, Huso D, Lowenstein CJ: P53-induced microRNA-107 inhibits HIF-1 and tumor angiogenesis. Proc Natl Acad Sci 2010, 107:6334-6339.

59. Del Bo R, Scarlato M, Ghezzi S, Martinelli Boneschi F, Fenoglio C, Galbiati S, Virgilio R, Galimberti D, Galimberti G, Crimi M, Ferrarese C, Scarpini E, Bresolin N, Comi GP: Vascular endothelial growth factor gene variability is associated with increased risk for AD. Ann Neurol 2005, 57:373-380.

60. Bai S, Nasser MW, Wang B, Hsu SH, Datta J, Kutay H, Yadav A, Nuovo G, Kumar P, Ghoshal K: MicroRNA-122 inhibits tumorigenic properties of pepatocellular carcinoma cells and sensitizes these cells to sorafenib. J Biol Chem 2009, 284:32015-32027.

doi:10.1186/1471-2350-13-35

Cite this article as: Augustin et al: Computational identification and experimental validation of microRNAs binding to the Alzheimer-related gene ADAM10. BMC Medical Genetics 2012 13:35. 